\title{
Resistance to rolling in the adhesive contact of two elastic spheres
}

\author{
By C. Dominik and A. G. G. M. Tielens \\ NASA Ames Research Center, Mail-Stop 245-3, Moffett Field, CA 94035, USA
}

[Received 20 December 1994 and accepted 21 February 1995]

\begin{abstract}
For the stability of agglomerates of micron sized particles it is of considerable importance to study the effects of tangential forces on the contact of two particles. If the particles can slide or roll easily over each other, fractal structures of these agglomerates will not be stable. We use the description of contact forces by Johnson, Kendall and Roberts, along with arguments based on the atomic structure of the surfaces in contact, in order to calculate the resistance to rolling in such a contact. It is shown that the contact reacts elastically to torque forces up to a critical bending angle. Beyond that, irreversible rolling occurs. In the elastic regime, the moment opposing the attempt to roll is proportional to the bending angle and to the pull-off force $P_{c}$. Young's modulus of the involved materials has hardly any influence on the results. We show that agglomerates of sub-micron sized particles will in general be quite rigid and even long chains of particles cannot be bent easily. For very small particles, the contact will rather break than allow for rolling. We further discuss dynamic properties such as the possibility of vibrations in this degree of freedom and the typical amount of rolling during a collision of two particles.
\end{abstract}

\section{$\$ 1$ INTRODUCTION}

The coagulation of small solid particles is a process of great importance in a wide range of fields such as material sciences (sintering) atmospheric sciences (aerosols) and also astrophysics (the formation of planets; see, for example, Weidenschilling, Donn and Meakin (1988)). Many studies of coagulation have been carried out both on the experimental side and on the theoretical side. Coagulated aggregates have structures that vary from quite compact to open fractal. Numerical simulations and also theoretical arguments (see, for example, Ball and Witten (1984)) have shown that the density of aggregates reflects the formation process, in particular the type of trajectory of the approaching particles (ballistic or random walk). A further important factor is the difference between particle-cluster aggregation and cluster-cluster aggregation. In particular, the addition of single particles to the cluster gives rise to quite compact structures as compared to cluster-cluster aggregation.

However, examination of pictures of (especially low density) aggregates shows that the stability of the adhesive joints is also an important factor for the structure (Kantor and Witten 1984). Often, the aggregates consist mainly of quasi-linear chains of particles with no more than two contacts per particle. Only strong joints can survive collisions with further particles without tearing, and only if the joints can resist tangential forces will the chains continue to be linear. If, for example, rolling of one grain over the other were easy, the chains would quickly fold and the aggregates would become considerably more compact. 
As far as the breaking of the aggregate is concerned, certainly the most important parameter is the pull-off force

$$
P_{\mathrm{c}}=3 \pi \gamma R,
$$

which is the force required to separate two spherical particles in an adhesive contact. Here $2 \gamma$ is the specific energy of adhesion for the two surfaces and $1 / R=1 / R_{1}+1 / R_{2}$ is the reduced radius of the particles involved. The factor 3 is the value obtained from the JKRS theory (see below). For very hard spheres, this factor should rather be 4 (Muller, Yushenko and Derjaguin 1982).

When the forces involved are not sufficiently large to destroy an aggregate, it is still possible to deform it by bending the particle chains, rolling the spheres over each other or sliding the surfaces along each other. The latter is not likely to be too important after the formation of the aggregate, since the forces needed to break a contact by horizontal forces are usually larger than the pull-off force (Savkoor 1992). However, what should be much easier is bending these chains, and eventually moving the contacts by rolling the particles over each other.

To get a handle on this, it is important to know which forces oppose the attempt to roll one body over the other in an adhesive contact. In this paper, we will develop a model which invokes both the properties of an adhesive contact between two elastic spheres and, to some extent, the molecular structure of the surfaces in order to calculate these forces and to estimate the energy dissipated in the rolling process.

\section{$\S 2$. THE JKRS SOLUTION FOR THE CONTACT PROBLEM BETWEEN TWO ADHESIVE SPHERES}

Our considerations are based to a large extent on the solution of the adhesive contact problem which was developed by Johnson, Kendal and Roberts (1971) (JKR) and also by Sperling (1964). The JKRS solution is based on fracture-like energy considerations. The main assumption leading to this solution is that the forces between the two spheres are pure contact forces. That is, two grains feel only an attractive forces across the contact area, while the surface areas outside the actual contact area are force-free. Certainly, such an approximation has its limitation. It was shown by Muller, Yushenko and Derjaguin (1979) that JKRS is more appropriate in the case of large soft spheres. For small and very hard spheres, the description by Derjaguin, Muller and Toporov (1975) is more accurate. We have decided to adopt JKRS for the time being, since the assumption of contact forces considerably simplifies the discussion of rolling resistance.

JKR showed that two elastic adhesive spheres make contact over a finite circular region, as indicated in fig. 1 . In the case of no external applied forces, the radius of this contact area is

$$
a_{0}=\left(\frac{9 \pi \gamma R^{2}}{E^{*}}\right)^{1 / 3}
$$

where $\gamma$ is the surface energy of each surface. For different surfaces, $\gamma=\gamma_{1}+\gamma_{2}-2 \gamma_{12}$, where $\gamma_{12}$ is the interface energy. $R$ is the reduced radius of the two spheres $\left(R^{-1}=R_{1}^{-1}+R_{2}^{-1}\right)$ and $\left(E^{*}\right)^{-1}=\left(1-v_{1}^{2}\right) / E_{1}+\left(1-v_{2}^{2}\right) / E_{2}$, where $E_{i}$ and $v_{i}$ are Young's modulus and Poisson's ratio, respectively, of grain $i$. The elastic displacement of the two spheres along the line connecting the centres of the two spheres (again with 
Fig. 1

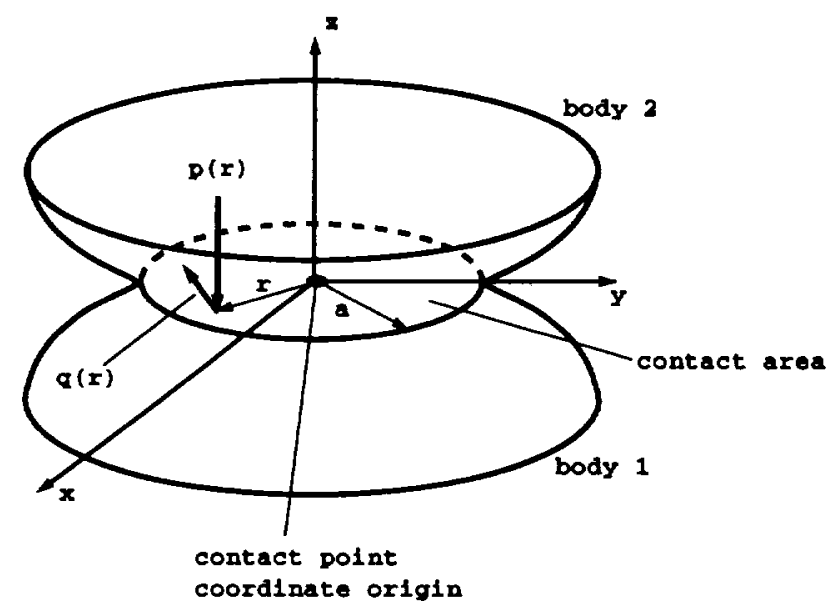

Sketch of the geometry of the contact area between two elastic spheres. The two bodies make contact over a circular region with radius $a$. Inside the contact region, the surfaces interact by a mutual pressure $p$ (which in adhesive contact can also be negative). When tangential forces are applied, they are transmitted by tangential tractions $q$ in the contact area. The contact area is always in the $x-y$ plane.

zero external force) is given by

$$
\delta_{0}=\frac{P_{\mathrm{c}}}{E^{*} a_{0}}
$$

where $-P_{\mathrm{c}}=-3 \pi \gamma R$ is the maximum external force that can be applied without breaking the contact (the so-called pull-off force). In the following we will sometimes use dimensionless quantities, denoted by a hat over a symbol:

$$
\hat{P}=\frac{P}{P_{\mathrm{c}}}, \quad \hat{\delta}_{z}=\frac{\delta_{z}}{\delta_{0}}, \quad \hat{a}=\frac{a}{a_{0}} .
$$

Here $P$ is the applied external load. When this load differs from zero, both the contact radius and the displacement change. The following relations between load, displacement and contact radius hold for the JKRS solution:

$$
\begin{aligned}
& \hat{P}=4\left(\hat{a}^{3}-\hat{a}^{3 / 2}\right), \\
& \hat{\delta}=3 \hat{a}^{2}-2 \hat{a}^{1 / 2} .
\end{aligned}
$$

The distribution of pressure in the contact area is given by a superposition of Hertz's and Boussineq's contact problems:

$$
p(r, a)=p_{\mathrm{H}}\left(1-r^{2} / a^{2}\right)+p_{\mathrm{B}}\left(1-r^{2} / a^{2}\right)^{-\mathrm{I} / 2} .
$$

In JKRS, the two constants are given by

$$
\begin{aligned}
& p_{\mathrm{H}}=6 \frac{P_{\mathrm{c}}}{\pi a_{0}^{2}} \hat{a}, \\
& p_{\mathrm{B}}=-2 \frac{P_{\mathrm{c}}}{\pi a_{0}^{2}} \hat{a}^{-1 / 2} .
\end{aligned}
$$




\section{\$3. RESistance TO ROLLING IN A JKRS CONTACT}

Resistance to rolling is a force that opposes the attempt to roll a body on the surface of another body. To measure this force we might apply a force $Q_{x}$ at the centre of a sphere with radius $R_{1}$ in the $x$ direction parallel to surface on which the sphere rolls.

In order to study this effect independently of the sliding process, we assume that sliding friction is high enough to prevent sliding entirely. Then, the tangential force $Q_{x}$ is balanced by an equal force in the contact area. However, since the two forces are applied at different points on the sphere, there is a resulting torque about the $y$ axis which is

$$
M_{y}=Q_{x} R \text {. }
$$

In order to prevent the sphere from starting to roll, this torque has to be balanced by an appropriate pressure distribution in the contact area. In order to produce a resultant moment (the so-called rolling moment), the distribution would have to be asymmetric with respect to the $y$ axis. However, the pressure distribution in JKRS is always cylindrically symmetric with respect to the contact point. Since this implies $p(x, y)=p(-x, y)$, there is no moment associated with this distribution:

$$
M_{y}=\iint x p(x, y) \mathrm{d} x \mathrm{~d} y=0 .
$$

Different mechanisms have been discussed in order to explain the observed rolling friction at least in the adhesion-free problem. The different possibilities are micro-slip at the interface, ineleastic deformation of the involved materials and surface irregularities; these have been summarized by Johnson (1989). However, none of these mechanisms should be of great importance in the contact of (sub-) micron-sized particles, of which adhesive aggregates usually consist. The displacements at the surfaces of the particle are usually not much larger than one or a few diameters of the atoms in the solid. Therefore, the movement of dislocations, which for macroscopic crystalline bodies is the source of inelastic behaviour, will play no role at very small particle sizes. Likewise, surface roughness is unimportant on the $50 \AA$ size scale of the contact area of sub-micron-sized particles.

However, for very small spheres, the surfaces can no longer be treated as perfectly smooth at all scales, since they are made of atoms. Then it becomes clear that the contact area cannot be perfectly symmetric. During rolling, new contact at the leading edge of the contact will not be made continuously, but rather in steps after a rolling distance of approximately one grid constant of the material. Likewise, at the trailing edge, the contact is lost in steps. Therefore the pressure distribution will not be exactly symmetrical all the time.

Still, if new contacts at the leading edge are made very smoothly and if the processes at the leading and trailing edges are exactly time reversed to each other, no energy will be dissipated and the pressure distribution will still be symmetric on average. This means that at every instant there may be a non-vanishing torque, but this torque may change sign and the average will still be zero. However, it is known from experiments with the atomic force microscope (Landman, Luedtke, Burnham and Colton 1990) that the approach of a tip to an adhesive surface and subsequent retraction involves a hysteresis. Upon close approach, the tip will form an adhesive contact with the substrate. Upon retraction, however, the tip will pull out a neck of surface material and contact will not be broken until a critical displacement is reached. A similar process occurs for a single atom attracted by van der Waals forces to the substrate and held back by 
Fig. 2

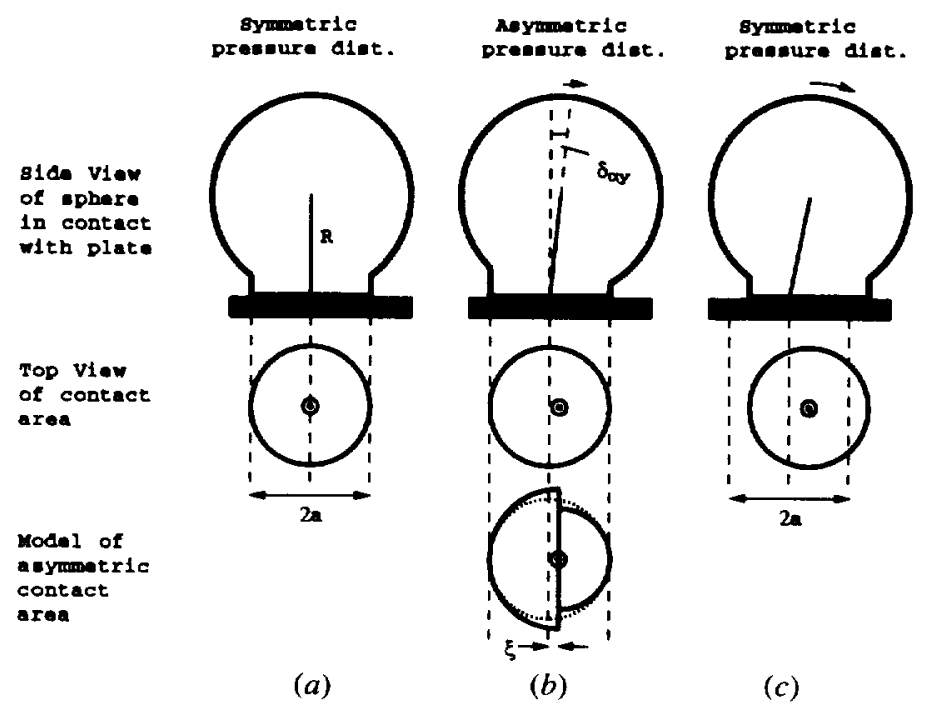

Sketch of the mechanism of rolling friction. Top: side-view on an elastic sphere in adhesive contact with a rigid plane. Centre: top-view on the contact area. The dotted circle indicates the position of the contact point (the projection of the sphere centre). From left to right, the diagram shows $(a)$ an initial torque-free state with contact area centred around the contact point, $(b)$ a state with resulting torque where the contact area is not centred and $(c)$ the final and again torque-free state after the contact area has readjusted. Bottom: decomposition of the asymmetric contact area into two half-circles of different radii.

spring-like binding forces in the approaching tip (see, for example, the discussion in Israelachvili (1992)). Upon approach, the atom will jump into contact with the substrate. The energy difference between the two states before and after the jump is dissipated. The same process occurs upon retraction between a different pair of states. It has been suggested by Tománek (1993) that this hysteresis might be the source of atomic scale rolling friction. In this paper, we follow up this idea and try to calculate the forces involved.

A possible approach to calculate the rolling friction in an adhesive contact would be to look directly at the energy dissipated in the making and breaking of contacts. This, however, requires an accurate knowledge of the forces between the atoms and an accurate description of the transition between the contact area itself and the surrounding parts of the spheres.

We therefore follow a different approach. The retarded making and breaking of contacts means that the contact area will not always be centred around the line connecting the centres of the two spheres, but rather will be shifted by a certain amount to one side. This shifted contact area leads to an asymmetric pressure distribution, as we will show below.

For now we restrict out terminology to the case of a rolling elastic sphere on a rigid plane. However, the case of two elastic spheres can be treated by simply replacing the sphere radius $R$ and the elastic constant $E^{*}$ with reduced quantities.

To describe the rolling process we visualize it as shown in fig. 2 .

(1) We start from a situation where the contact is a JKRS contact centred around 
the contact point which is the point vertically below the centre of the sphere. With two spheres it would be the point on the line connecting the two centres.

(2) Then the sphere is rolled a small distance over the surface. If $\delta_{x y}$ denotes the angular motion, the contact point moves a distance $\xi=R \delta_{\alpha y}$. However, we assume that the contact area remains behind. Thus the contact area is no longer centred around the actual contact point, but is shifted by a distance $\xi$. In this state there will be a non-vanishing torque opposing the rolling direction. In $\$ 3.1$ we will calculate this torque.

(3) At a certain critical rolling distance $\xi^{\text {yield }}$ (which should be typically of the order of one atom diameter in the material) the contact area readjusts - that is, contact is lost at the trailing edge and new contact is formed at the leading edge-so that again a symmetrical (torque-free) distribution is formed.

In the following we will calculate the moment that is produced by the asymmetric pressure distribution.

\subsection{The moment associated with a shifted contact area}

To our knowledge, there are no analytical solutions for the problem of a shifted contact area. To calculate the pressure distribution and the associated moment we proceed as follows:

(1) The true shifted contact area is approximated by a decomposition into two half circles of different radii $a+\xi$, respectively (see fig. 2 ).

(2) The half circle $x<0$ is one half of a symmetrical contact with contact radius $a+\xi$ and pressure distribution $p\left(r, a+\xi, \delta_{z}\right)$ (see below for the definition of this pressure distribution).

(3) the half circle $x>0$ has a smaller contact radius $a-\xi$ and a corresponding pressure distribution $p\left(r, a-\xi, \delta_{z}\right)$.

(4) The total momentum due to both half circles is calculated.

The resulting distribution is discontinuous at $x=0$. As indicated in fig. 2 , the true contact area is closely fitted far from the $y$ axis where the contributions to the moment are most important. In Appendix 1 we discuss the two-dimensional (2D) case where the exact pressure distribution can be obtained analytically. That analysis shows that the above approximation gives reasonable results.

We start from the general pressure distribution for the contact of two elastic spheres with a contact radius $a$. This pressure distribution is the superposition of the solutions of the Hertz problem and of the Boussineq problem. The subscripts $\mathrm{H}$ and $\mathrm{B}$ indicate that in the following:

$$
p\left(r, a, \delta_{z}\right)=p_{\mathrm{H}}\left(1-r^{2} / a^{2}\right)^{1 / 2}+\frac{p_{\mathrm{B}}}{\left(1-r^{2} / a^{2}\right)^{1 / 2}} .
$$

The pressure distribution corresponds to a normal displacement of the spheres

$$
\delta_{z}=\frac{\pi a}{2 E^{*}}\left(p_{\mathrm{H}}+2 p_{\mathrm{B}}\right)
$$

To calculate the moments of one half of the contact area for different sizes for the contact area we cannot directly use the JKRS equations since the different contact radii $a$ always correspond to different values for $\delta_{z}$. For the intended calculation, however, 
we need contact areas of different sizes but with the same normal displacement $\delta_{z}$. We have thus to start from eqn. (12).

For a contact area of a given size, the constant $p_{\mathrm{H}}$ has the fixed value $p_{\mathrm{H}}=2 a E^{*} / \pi R$, since it is this part of the solution that deforms the spherical surface to a flat area. Different values of $\delta_{z}$ can be achieved by changing only the Boussineq part of the solution. Therefore we will now replace the Boussineq constant $p_{B}$ with eqn. (13). Then, we may keep $\delta_{z}$ constant and change the size of the contact area.

An expression for $p_{B}$ can be obtained from eqn. (13):

$$
p_{\mathrm{B}}(a)=\frac{E^{*}}{\pi a} \delta_{z}-\frac{1}{2} p_{\mathrm{H}}(a) .
$$

We insert this into eqn. (12) and get

$$
p\left(r, a, \delta_{z}\right)=p_{\mathrm{H}}\left(1-r^{2} / a^{2}\right)^{1 / 2}+\frac{\left(E^{*} / \pi a\right) \delta_{z}-p_{\mathrm{H}} / 2}{\left(1-r^{2} / a^{2}\right)^{1 / 2}} .
$$

As discussed above, we compose the asymmetric contact region of two halves. The distribution inside each half is taken to be that of a symmetric pressure distribution with contact radius $a+\xi$ and $a-\xi$, respectively:

$$
p_{a}(r, a, \xi)= \begin{cases}p\left(r, a+\xi, \delta_{z}\right), & x<0, \\ p\left(r, a-\xi, \delta_{z}\right), & x>0\end{cases}
$$

The moment associated with this distribution is

$$
M_{y}=\int_{\pi / 2}^{3 \pi / 2} \int_{0}^{a+\xi} x p\left(r, a+\xi, \delta_{z}\right) r \mathrm{~d} r \mathrm{~d} \varphi+\int_{-\pi / 2}^{\pi / 2} \int_{0}^{a-\xi} x p\left(r, a-\xi, \delta_{z}\right) r \mathrm{~d} r \mathrm{~d} \varphi .
$$

With $x=r \cos \varphi$ we may carry out the $\varphi$ integration and find

$$
\begin{aligned}
M_{y} & =-2 \int_{0}^{a+\xi} r^{2} p\left(r, a+\xi, \delta_{z}\right) \mathrm{d} r+2 \int_{0}^{a-\xi} r^{2} p\left(r, a-\xi, \delta_{z}\right) \mathrm{d} r \\
& =-M_{y, a+\xi}^{(1 / 2)}+M_{y, a-\xi .}^{(1 / 2)} .
\end{aligned}
$$

Thus we have to solve integrals of the kind

$$
M_{y, \mathscr{A}, \delta_{z}}^{(1 / 2)}=2 \int_{0}^{\mathscr{A}} r^{2} p\left(r, \mathscr{A}, \delta_{z}\right) \mathrm{d} r .
$$

The required integrals are given by eqns. (87) and (88) in Appendix B. The result is

$$
\begin{aligned}
M_{y, \mathscr{A}, \delta z}^{(1 / 2)} & =2\left[p_{\mathrm{H}} \frac{\pi}{16} \mathscr{A}^{3}+\left(\frac{E^{*}}{\pi \mathscr{A}^{2}} \delta_{z}-\frac{1}{2} p_{\mathrm{H}}\right) \frac{1}{4} \mathscr{A}^{3}\right] \\
& =\frac{1}{2} E^{*} \mathscr{A}^{2} \delta_{z}-\frac{\pi}{8} p_{\mathrm{H}} \mathscr{A}^{3} .
\end{aligned}
$$

We insert the correct value of $p_{\mathrm{H}}$ (eqn. (8)):

$$
M_{y, \mathscr{A}, \delta_{z}}^{(1 / 2)}=\frac{E^{*}}{2} \delta_{z} \mathscr{A}^{2}-\frac{3}{4} P_{\mathrm{c}} \frac{\mathscr{A}^{4}}{a_{0}^{3}} .
$$

The moment of the whole asymmetric contact can now be calculated from eqn. (19). We keep $\delta_{z}$ constant and neglect all terms of higher than first order in $\xi / a$. This is equivalent to the substitutions $\mathscr{A}^{4} \rightarrow-8 a^{3} \xi$ and $\mathscr{A}^{2} \rightarrow-4 a \xi$ : 


$$
M_{y} \approx 2 \xi\left(-E^{*} a \delta_{z}+3 P_{\mathrm{c}} \hat{a}^{3}\right) .
$$

Now we may insert the correct value $\delta_{z}$ which is the value from Johnson's solution corresponding to a contact area radius $a$ as given in eqn. (6). The result is

$$
M_{y}=4 P_{c} \hat{a}^{3 / 2} \xi .
$$

The moment associated with a contact area shifted by a distance $\xi$ is therefore proportional to the pull-off force and to the shift itself. The order of magnitude of this result is what one would have expected, since the forces involved are of the order of $P_{\mathrm{c}}$ and the force is applied over a level of length $\xi$. The dependence on the normal load $P$ enters through the factor $\hat{a}^{3 / 2}$, which should be close to one in most cases (see below).

In the following section we will discuss in greater detail the properties of the rolling moment and its implications on the structure and stability of agglomerates.

\section{§ 4. MechaniCal PROPERTIES OF THE CONTACT UNDER THE INFLUENCE OF ROLLING MOMENTS}

\subsection{Static considerations}

Equation (25) shows that the force opposing the attempt to roll one particle over the other is a torque that is proportional to the angular displacement. However, as the displacement increases there is a limit to the increase in that force. When the line connecting the two particle centres has moved a certain critical distance over the surfaces, the contact area will start to move, and the force will decrease or drop to zero. We will call this critical distance the yield displacement $\xi^{\text {yield }}=R \delta_{\alpha}^{\text {yield }} . \xi^{\text {yield }}$ should be of the order of the distance between atoms in the materials, typically $2 \AA$. Therefore, the adhesive contact reacts elastically in a small displacement interval. For larger displacements, energy is dissipated and the contact area moves irreversibly over the surfaces.

Due to the simple structure of eqn. (25), the calculation of several mechanical properties is quite straightforward. We will illustrate the results in some figures, which show the involved forces and other quantities as functions of the surface energy $\gamma$ and of the particle radius $R$ (see figs. 3 and 6 ).

For the following discussion we will restrict ourselves to the contact between identical spheres with radius $R_{1}$. Then, $R=R_{1} / 2$ and $P_{\mathrm{c}}=\frac{3}{2} \pi \gamma R_{1}$. Furthermore, we neglect the variations in the rolling moment due to variations in the normal force. These enter into eqn. (25) in the factor $\hat{a}^{3 / 2}$. However, if the normal force stays within the range $-P_{c}<P<P_{c}$, the factor $\hat{a}^{1.5}$ varies only in the range between $0.5-1 \cdot 2$. Larger normal forces are likely to eventually destroy the contact, and are therefore not relevant to our problem. Therefore, we assume for most of the rest of the paper that $\hat{a}=1$.

Since we have neglected the normal load, the surface energy $\gamma$ is the sole material property that enters into the equations. In the diagrams, we will therefore usually plot $\gamma$ on the abscissa. Every material or combination of materials may then be associated with a position on this axis. We have plotted the positions of some materials on the top of the diagrams. The material properties used are summarized in the table. The ordinate of the plots is labelled by the reduced particle radius. Therefore, for a given system, the results can be read immediately from the figures.

The first quantity of interest is the tangential force that can be applied at the centre of the particles in order to exceed the yield displacement. In other words, to which limiting force does the contact still react elastically? A force $Q_{x}^{\text {yield }}$, applied at the particle centres, corresponds to an applied moment at the contact point of $M_{y}^{\text {yild }}=Q_{x}^{\text {yield }} R_{1}$, thus 
Fig. 3

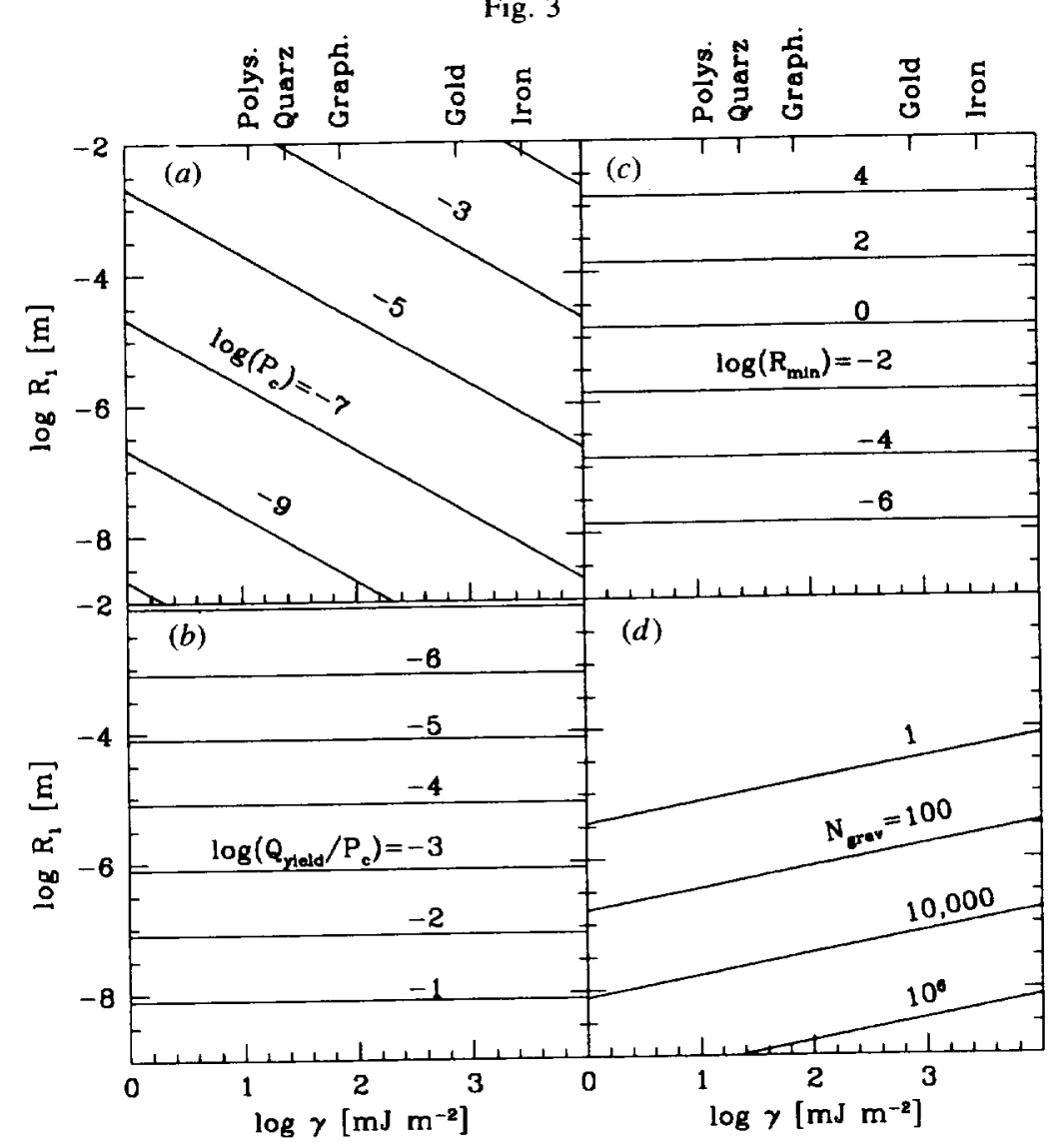

Plots of some results for the adhesive contact of identical spheres in the $\gamma-R_{1}$ plane. $(a)$ The pull-off force as calculated from JKRS $\left(P_{\mathrm{c}}=3 \pi \gamma R\right)$ (the labels marking the curves denote $\left.\log P_{\mathrm{c}}[\mathrm{N}]\right),(b)$ the yield force required to initiate rolling (see text) normalized by the pull-off force $Q_{\text {yield }} / P_{\mathrm{c}},(c)$ the minimum radius of curvature $\mathscr{R}_{\min }[\mathrm{m}]$ that can be applied to a linear chain without leaving the elastic domain, and $(d)$ the number $N_{\text {grav }}$ of particles that can be in a linear chain of particles in the earth's gravitational field without leaving the elastic domain. Calculated for particles with a mass density of $2 \mathrm{~g} \mathrm{~cm}^{-3}$.

$$
Q^{\text {yield }}=6 \pi \gamma \xi^{\text {yield }}
$$

or

$$
\frac{Q^{\text {yield }}}{P_{\mathrm{c}}}=4 \frac{\xi^{\text {yield }}}{R_{1}} \text {. }
$$

The absolute force $Q^{\text {yield }}$ needed to leave the elastic domain is independent of the particle radius, while the force in units of the pull-off force is independent of the surface energy $\gamma$. This behaviour is also reflected in the map of the normalized yield force in fig. $3(b)$. There it can be seen that for micron-sized or larger particles, the force required to initiate rolling is much smaller than the pull-off force. Therefore, it should be quite simple to roll particles of these sizes over each other, without immediately destroying the contact. On the other hand, for very small grains, the force needed to initiate rolling becomes comparable to the pull-off force. For particle radii of $1 \mathrm{~nm}$, the forces would even be the same-however, we have to bear in mind that at these sizes 
Material properties.

\begin{tabular}{lccccc}
\hline Material & $\gamma \dagger\left(\mathrm{mJ} \mathrm{m}^{-2}\right)$ & $E\left(\mathrm{~N} \mathrm{~m}^{-2}\right)$ & $v$ & $\rho\left(\mathrm{g} \mathrm{cm}^{-3}\right)$ & Reference \\
\hline Polystyrene & 12 & $3.4(9)$ & 0.5 & 1.04 & {$[2,3]$} \\
Quartz & $25 \ddagger$ & $5.4(10)$ & 0.17 & 2.6 & {$[1,2]$} \\
Graphite & 75 & $1 \cdot 0(10)$ & 0.32 & 2.2 & {$[4,5]$} \\
Gold & $790 \S$ & - & - & 19.3 & {$[7]$} \\
Iron & 3000 & $2 \cdot 1(11)$ & 0.27 & 7.7 & {$[2,6]$} \\
\hline
\end{tabular}

$\dagger$ Surface energy per surface.

$\ddagger$ Measured for micron-sized particles.

$\$$ Mean value of the range given in the reference.

References; [1] Kendall, Alford and Birchall 1987, [2] Anderson 1981, [3] Kendall and Padget 1987, [4] Brocklehurst 1977, [5] Zisman 1963, [6] Easterling and Thölen 1972, [7] Hodgman 1949.

the theory of elasticity is no longer applicable. The fact that both forces are comparable implies that any attempt to compress an aggregate by rolling the particles over each other would be likely to destroy the aggregate.

Another interesting quantity is the flexibility of particle chains. Many open aggregates mainly consist of quite linear chains in a tree-like structure. A way to treat this problem is to calculate the minimal radius of curvature that a linear chain of particles can adopt, again without leaving the elastic domain. If we bend every contact to its limit ( which is the angle $\delta_{\alpha}^{\text {yield }}=2 \xi^{\text {yield }} / R_{1}$ ), the radius of curvature of the chain is

$$
\mathscr{R}_{\text {min }}=\frac{R_{1}^{2}}{\xi^{\text {yield }}},
$$

a result that is mapped in fig. $3(\mathrm{c})$. The minimal radius of curvature is generally large compared to the radius of the particles. Even for rather small spheres $(100 \mathrm{~nm})$, the minimal radius of curvature is about $50 \mu \mathrm{m}$, thus a factor of 500 times larger. This means that a chain would have to be a few thousand grains long in order to be elastically bent to a circle. Chains shorter than this length would appear as quite rigid structures that could not be reversibly bent. Because of this rigidity, fractal 'fingers' of aggregates do not close up into ring-like structures easily.

In a laboratory, the aggregate is usually subjected to the earth's gravity. Another interesting question is, therefore, whether an aggregate will collapse under its own weight and flatten. We will restrict ourselves to a very simple consideration. We again look at a linear chain of $N$ particles bonded by adhesion and fixed at one end to the wall (c.f. fig. 4). We make the further simplification that the chain is not bent, but assume

Fig. 4

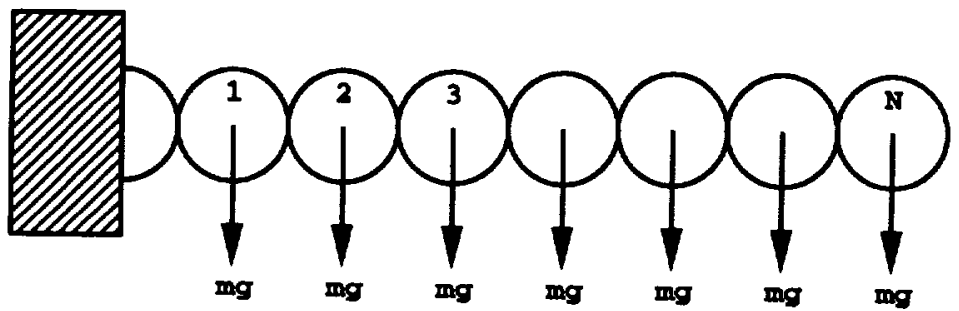

Experiment for the determination of the influence of the earth's gravitational field on the stability of an aggregate. 
that it is a straight line. This is certainly justified by the above discussion of the radius of curvature. We may then compute the torque that acts on the key contact of the chain (which is the strongest torque anywhere in the chain). It is given by

$$
M_{y}^{\text {grav }}(N)=m g R_{1} N^{2},
$$

where $m$ is the mass of an individual particle, $g$ is the gravitational acceleration and $N$ is the number of grains in the chain. Irreversible rolling occurs now when $N>N_{\text {grav }}$, where

$$
N_{\mathrm{grav}}=\left(\frac{9 \gamma \xi^{\text {yield }}}{2 R_{1}^{3} \rho g}\right)^{1 / 2}
$$

and $\rho$ is the specific density of the grain material. We have calculated $N_{\text {grav }}$ for $\rho=2 \mathrm{~g} \mathrm{~cm}^{-3}$ and plotted this in fig. 3 . The results hardly depend on the density, since $N_{\text {grav }}$ scales only with the square root of $\rho$. It is obvious that for particles larger than about $100 \mu \mathrm{m}$, even a single particle is heavy enough to start rolling under the earth's gravity. However, with decreasing particle size, the number of grains that can be supported increases rapidly. A chain of approximately one thousand $100 \mathrm{~nm}$ spheres is still stable under the earth's gravity. A more careful analysis of the stability of tenuous structures (not limited to straight chains of particles) was carried out by Kantor and Witten (1984), using a parametrized strength of the contact. The size limits established in their study are in good agreement with our results when appropriate estimates for the contact strength are used.

\subsection{Dynamic considerations}

\subsubsection{Vibrations}

As already noted above, for small angular displacements the moment produced by the displacement is proportional to the displacement itself. It should therefore be possible to excite vibrations where two particles in contact oscillate around the equilibrium state as shown in fig. $5(b)$. Before we calculate the frequency associated

Fig. 5

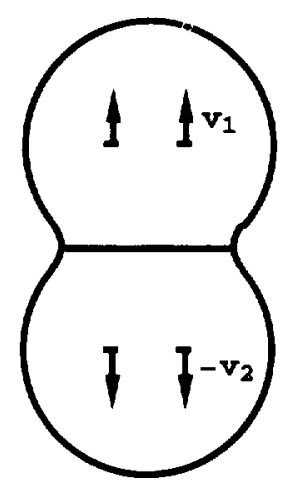

(a)

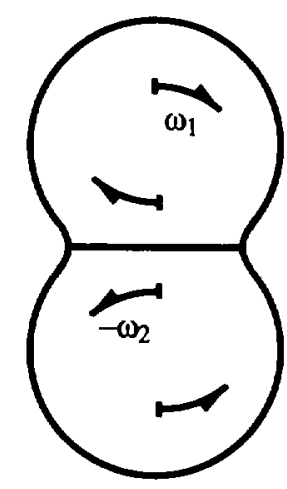

(b)

The two modes of vibration considered in $\$ 5.2 .1$. (a) Vertical vibration: the vertical displacement $\delta_{z}$ is oscillating, when the distance between the sphere centres increases and decreases; (b) rolling vibration: the angular displacement $\delta_{a y}$ oscillates, when the spheres roll back and forth over each other. 
with these oscillations, we will briefly discuss (as a means of comparison) a different type of vibration in which the particles oscillate along the $z$ axis as shown in fig. $5(a)$.

If $v_{z 1}$ and $v_{z 2}$ denote the velocities of the particles in the $z$ direction (defined as indicated in fig. 5), the equations of motion are

$$
\begin{aligned}
& m_{1} \dot{v}_{z 1}=P, \\
& m_{2} \dot{v}_{z 2}=-P .
\end{aligned}
$$

The change in the vertical displacement is given by $\dot{\delta}_{z}=v_{z 1}-v_{z 2}$, thus

$$
\ddot{\delta}=\dot{v}_{z 1}-\dot{v}_{z 2}=\frac{1}{m} P,
$$

where $m=\left(1 / m_{1}+1 / m_{2}\right)^{-1}$ is the reduced mass of the particles. $P$ is of course not a linear function of $\delta_{z}$, but we can linearize it in the vicinity of the equilibrium point $P=0$ (see also Thölen (1992)) and find

$$
\ddot{\delta}_{z} \approx \frac{6}{5} \frac{E^{*} a_{0}}{m} \delta_{z}
$$

The frequency of the vibrations is therefore given by

$$
\Omega_{z}^{0}=\frac{1}{2 \pi}\left(\frac{6}{5} \frac{E^{*} a_{0}}{m}\right)^{1 / 2}
$$

If we consider again identical spheres of radius $R_{1}$ and density $\rho$ and with $E_{1}^{*}=E_{1} /\left(1-v_{1}^{2}\right)$, we find

$$
\Omega_{z}^{0} \approx 6.38 \times 10^{8}\left(\frac{E_{1}^{*}}{10^{10} \mathrm{~N} \mathrm{~m}^{-2}}\right)^{1 / 3}\left(\frac{\gamma}{100 \mathrm{~mJ} \mathrm{~m}^{-2}}\right)^{1 / 6}\left(\frac{10^{-7} \mathrm{~m}}{R_{1}}\right)^{7 / 6}\left(\frac{2 g \mathrm{~cm}^{-3}}{\rho}\right)^{1 / 2} .
$$

Thus the frequencies are typically of the order of $\mathrm{GHz}$, and almost independent of the surface energy of the materials.

For the angular vibration we neglect all interactions with other degrees of vibration. The equations of motion then become

$$
\begin{aligned}
& m_{1} k_{1}^{2} \dot{\omega}_{1}=M_{y}\left(\delta_{x y}\right), \\
& m_{2} k_{2}^{2} \dot{\omega}_{2}=-M_{y}\left(\delta_{\alpha y}\right),
\end{aligned}
$$

where $\omega_{i}$ is the angular velocity of particle $i$ about the $y$ axis (as indicated in fig. (5)) and $k_{i}$ is the radius of gyration of the particle $\left((2 / 5)^{1 / 2}\right.$ times the radius for homogeneous spheres). The time derivative of the angular deformation is given by $\dot{\omega}_{1}-\dot{\omega}_{2}$, thus

$$
\begin{aligned}
\ddot{\delta}_{\alpha y} & =\dot{\omega}_{1}-\dot{\omega}_{2}=\left(\frac{1}{m_{1} k_{1}^{2}}+\frac{1}{m_{2} k_{2}^{2}}\right) M_{y}=\frac{1}{I} M_{y} \\
& =\frac{4 P_{c} R}{I} \delta_{\alpha y},
\end{aligned}
$$

where $I$ is the reduced moment of momentum of the two spheres. Thus, the system oscillates in this degree of freedom with an eigenfrequency of

$$
\Omega_{\mathrm{roll}}^{0}=\frac{1}{2 \pi}\left(\frac{4 P_{\mathrm{c}} R}{I}\right)^{1 / 2} .
$$


Fig. 6

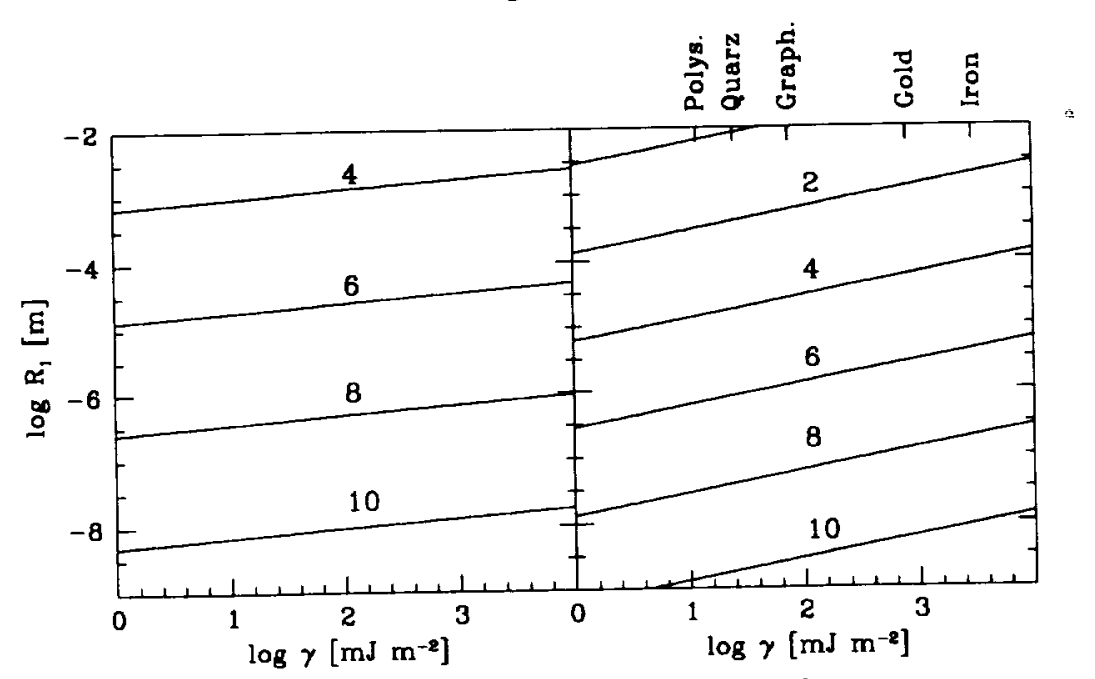

(a)

(b)

Eigenfrequencies for the $z$ vibration $(a)$ and the rolling vibration $(b)$ for two identical spheres in contact, plotted as contour lines in a radius-surface tension diagram. The density of the materials was assumed to be $\rho=2 \mathrm{~g} \mathrm{~cm}^{-3}$. In $(a)$ the quantity $E_{1}^{*}$ (see text) was set to $10^{10} \mathrm{~N} \mathrm{~m}^{-2}$. The curves are labelled by $\log$ (frequency) in units of $\mathrm{Hz}$.

Considering two identical homogeneous spheres with radii $R_{1}$ and a mass density, $\rho$, we have

$$
\begin{aligned}
R & =R_{1} / 2, \quad k_{1}=k_{2}=(2 / 5)^{1 / 2} R_{1}, \quad m_{1}=m_{2}=(4 / 3) \pi R_{1}^{3} \rho, \\
\Omega_{\text {roll }}^{0} & =\frac{3}{2 \pi}\left(\frac{\gamma}{5 R_{1}^{3} \rho}\right)^{1 / 2} \\
& \approx 4.77 \times 10^{7}\left(\frac{\gamma}{100 \mathrm{~mJ} \mathrm{~m}^{-2}}\right)^{1 / 2}\left(\frac{10^{-7} \mathrm{~m}}{R_{1}}\right)^{3 / 2}\left(\frac{2 \mathrm{~g} \mathrm{~cm}^{-3}}{\rho}\right)^{1 / 2} .
\end{aligned}
$$

These frequencies as functions of $\gamma$ and $R_{1}$ are shown in fig. 6. The eigenfrequency associated with the rolling motion is usually one to two orders of magnitude smaller than the frequency of the vertical vibration.

\subsubsection{Energy dissipation during rolling}

The rolling vibration can only take place if the amplitude is small enough. For amplitudes larger than a critical angle $\delta_{x y}^{\text {yield }}=\xi^{\text {yield }} / R$, the spheres start rolling irreversibly over each other. When we assume that after a rolling distance $\xi^{y i e l d}$ the contact area readjusts to a symmetric contact with zero torque, the average torque is (owing to the linear dependence between torque and $\xi$ )

$$
\left\langle M_{y}\right\rangle \approx 2 P_{c} \xi^{\text {yield }}
$$

which is equivalent to an average decelerating force applied at the centre of the rolling sphere of

$$
\left\langle Q_{x}\right\rangle \approx 2 P_{\mathrm{c}} \frac{\xi^{\text {yield }}}{R}
$$


Fig. 7

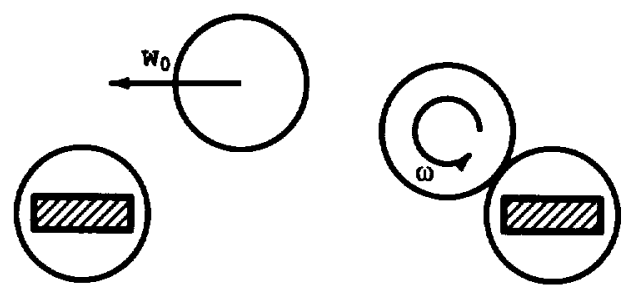

Experiment to show the amount of energy lost due to rolling friction. One of the two spheres is fixed in an inertial system (hatched area). The other sphere is approaching with a kinetic energy $W_{0}$. Upon contact, the energy $W_{0}$ is distributed between rotation and linear movement. The moving sphere rolls over the fixed sphere until all energy is dissipated.

In order to visualize this energy loss we will calculate the number of full rotations a sphere can roll with a given energy at the start. We consider a sphere colliding with a fixed sphere (see fig. 7). We assume that sliding friction is strong enough to entirely prevent sliding, so that the surfaces do not move relative to each other. Therefore, the initial energy $W_{0}$ is distributed into rolling and linear motion:

$$
W_{0}=\frac{1}{2} m_{1} v_{1}^{2}+\frac{1}{2} l_{1} \omega_{1}^{2}
$$

Since no sliding occurs, $v_{1}=\omega_{1} R_{1}$. If we further observe that for a homogeneous sphere $I=\frac{2}{5} m_{1} R_{1}^{2}$, we find that the rotation of the sphere just after contact is

$$
\omega(t=0)=\left(\frac{4}{7} \frac{W_{0}}{I_{1}}\right)^{1 / 2}
$$

From then on the equation of motion $\omega_{1}$ is given by

$$
\dot{\omega}_{1}=-\frac{M_{y}}{I_{1}}
$$

where $M_{y}$ is given by eqn. (43). Integrating eqn. (47) we can find the time when the rotation of the sphere stops, and calculate the number of rotations performed. The result is

$$
n_{\text {roll }}=\frac{1}{14 \pi} \frac{W_{0}}{P_{c} \xi^{\text {yield }}} .
$$

Thus, the number of rotations is proportional to the initial energy and inversely proportional to the pull-off force. This seems to indicate that rolling is much more likely in collisions between grains with a small pull-off force. However, it is also to be recognized that grains with a small pull-off force will only stick at small impact energies. We should therefore measure the energy $W_{0}$ in units of an energy typical for adhesion. Such an energy is given by $P_{c} \delta_{0}$. It has been shown by Chokshi, Tielens and Hollenbach (1993) that this energy is of the order of the maximum energy that can be dissipated in a slow collinear collision between two spheres, so that they will stick together. Then, eqn. (48) becomes

$$
n_{\text {roll }}=\frac{3}{14(9 \pi)^{1 / 3}}\left(\frac{\gamma}{E^{*}}\right)^{2 / 3} \frac{R^{1 / 3}}{\xi^{\text {yield }}}\left(\frac{W_{0}}{P_{\mathrm{c}} \delta_{0}}\right) .
$$

Thus the material properties enter only in the form of the ratio $E^{*} / \gamma$ and we may plot $n_{\text {roll }}$ in a map as shown in fig. 8. Even in a collision with relatively large energies 
Fig. 8

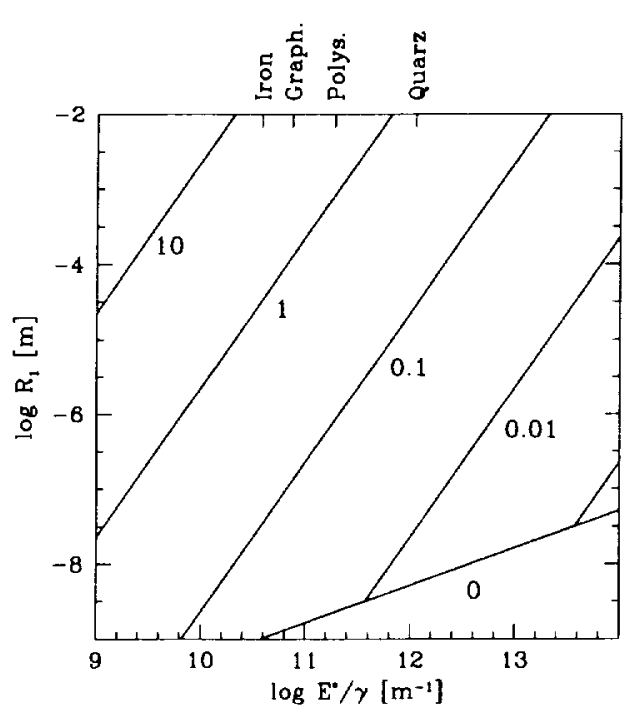

Plot of the number of rotations a mobile sphere rolls over a fixed sphere with an initial tangential kinetic energy $P_{\mathrm{c}} \delta_{\mathrm{D}}$. The curves are labelled by $n_{\text {roll }} /\left(W_{0} / P_{\mathrm{c}} \delta_{0}\right)$. In the region to the lower right, the rolling distance is smaller than $\xi^{y i e l d}$.

$\left(W_{0} \approx P_{\mathrm{c}} \delta_{0}\right.$ ), spheres with a large ratio $E^{*} / \gamma$ do not roll at all, if they are smaller than about $10 \mathrm{~nm}$. Larger radii, smaller elastic moduli, or larger surface energies are favourable to more rotation-however, the rotation is usually less than one complete rotation if the radius of the spheres stays below $100 \mathrm{~nm}$. For bigger spheres, it becomes quite possible that rolling persists for a full rotation or more. This means that in a collision, the contact would not remain at the point of first contact. Rather, the spheres will roll over each other. If the fixed sphere is part of an aggregate, the mobile sphere will probably roll until at least a second point of contact is established. Therefore, the resulting aggregates will be considerably more compact.

\section{$\S 5$. CONCLUSIONS}

In this paper we have discussed the resistance of a JKRS contact to rolling. This was done by recognizing that, due to the atomic structure of the surfaces on contact, the contact area will not be symmetric around the contact point. This asymmetric contact area leads to a net torque force opposing the attempt to roll one body over the other. We have calculated the torque of the asymmetric contact area by an approximation in which the area was divided into two half cycles of different size. This approximation was justified by an exact solution for the two-dimensional case. However, it would be very interesting to see an exact analytical solution for the three-dimensional case.

Since the maximum asymmetry of the contact area is given by the typical length in the atomic grids of the bodies irreversible rolling starts only when the bodies roll at least this critical distance. For smaller distances, the contact reacts elastically with a force proportional to the displacement and to the pull-off force. As long as the contact is not subjected to normal forces, the resistance to rolling is dependent on the surface energy alone. Normal forces introduce a very weak dependence upon Young's modulus.

We have also discussed some implications of our results, many of which should be testable by experiments. The results indicate a critical bending angle below which the 
bending will be elastic. The critical angle is quite small, so that long linear chains of particles would appear rather rigid. We also showed that in the case of very small particles the force needed to bend a chain can be comparable with the pull-off force. Therefore, aggregates of very small grains would be very stiff. Forces applied to these aggregates are likely to break them instead of deforming them considerably.

Finally we have shown that it should in principle be possible to excite vibrations not only in the vertical degree of freedom, but also in the rolling degree of freedom. This should be visible in vibrations of fractal aggregates as well.

\section{ACKNOWLEDGMENTS}

One of the authors (C.D.) was supported during this work by the National Research Council. Theoretical studies of interstellar dust at NASA Ames is supported under task 399-20-01-30 through NASA's Theory Program.

\section{APPENDIX A}

COMPARISON OF EXACT AND SIMPLIFIED CALCULATION OF THE ROLLING MOMENT OF AN ASYMMETRIC ADHESION PROFILE

In this appendix we will calculate the pressure distribution and the associated rolling moments of an elastic cylinder in contact with a rigid plane. In this $2 \mathrm{D}$ case, it is possible to calculate the pressure distribution in an asymmetric contact analytically. This will enable us to compare the rolling moment obtained from a simplifying assumption as used in $\$ 4.1$ with an exact solution.

Although we here describe only a contact between an elastic cylinder and a rigid plane, the results can be immediately generalized to the case of two elastic aligned cylinders by just replacing the cylinder radius $R$ and the elastic constant $E^{*}=E /\left(1-v^{2}\right)$ with reduced values. As usual, $E$ is the elastic modulus of the material and $v$ is the Poisson's ratio.

Both the symmetric and the asymmetric contact are shown in fig. 9. Our treatment of this 2D contact problem follows the description by Johnson (1989).

\section{A.1. The symmetric pressure distribution}

When the cylinder is in contact with the plane, the deformation $u_{z}$ of the surface of the cylinder is given by

$$
u_{z}(x)=\delta_{z}-\frac{x^{2}}{2 R}, \quad-a \leq x \leq a,
$$

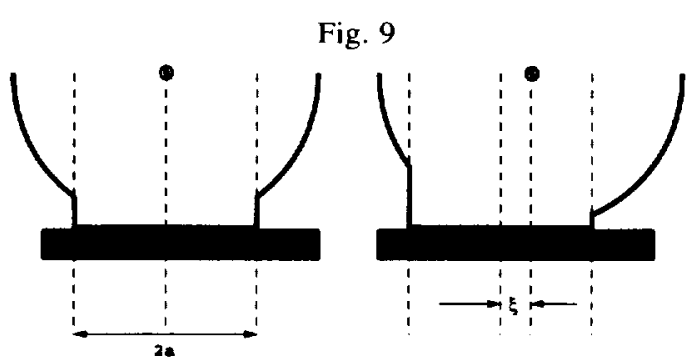

Symmetric and asymmetric contact of a cylinder with a rigid plane. 
where we put the origin of the coordinate to the same $x$ coordinate as the centre of the cylinder. In the case of specified deformation in the loaded region, the pressure distribution $p(x, a)$ can be calculated from the following integral equation (see, for example, Johnson (1989)):

$$
\int_{-a}^{a} \frac{p(s, a)}{x-s} \mathrm{~d} s=-\frac{\pi E}{2} \frac{\partial u_{z}}{\partial x}
$$

This is an integral equation of the type

$$
\int_{-a}^{a} \frac{p(s, a)}{x-s} \mathrm{~d} s=g(x)
$$

The general solution of this equation can be obtained by applying the finite Hilbert transformation which leads to

$$
p(x, a)=-\frac{1}{\pi^{2}\left(a^{2}-x^{2}\right)^{1 / 2}}\left[\int_{-a}^{a} \frac{\left(a^{2}-s^{2}\right)^{1 / 2} g(s)}{x-s} \mathrm{~d} s+C\right] .
$$

where $C$ is some constant of integration. In the following we add a subscript $s$ to the pressure distribution in order to indicate that this is the symmetric case. The application of eqn. (53) to eqn. (51) yields

$$
\begin{aligned}
p_{\mathrm{s}}(x, a) & =-\frac{1}{\pi^{2}\left(a^{2}-x^{2}\right)^{1 / 2}}\left[C_{1}+\int_{-a}^{a} \frac{\pi E}{2 R} \frac{s\left(a^{2}-s^{2}\right)^{1 / 2}}{x-s} \mathrm{~d} s\right], \\
& =-\frac{E}{2 R\left(a^{2}-x^{2}\right)^{1 / 2}}\left[C_{2}+\frac{1}{\pi} \int_{-a}^{a} s \frac{\left(a^{2}-s^{2}\right)^{1 / 2}}{x-s} \mathrm{~d} s\right] .
\end{aligned}
$$

The integrand is singular at $x=s$ and we have to take the Cauchy principal value, which is given in eqn. (81). Inserting this result and rearranging yields

$$
p_{\mathrm{s}}(x, a)=\frac{E}{2 R}\left[\left(a^{2}-x^{2}\right)^{1 / 2}+\left(C-a^{2} / 2\right) \frac{1}{\left(a^{2}-x^{2}\right)^{1 / 2}}\right] .
$$

The first part of the pressure distribution is the usual Hertz solution for two cylinders pressed into contact. In this part of the solution the pressure falls to zero as $x$ goes to $a$. The second part which is proportional to $1 /\left(a_{2}-x^{2}\right)^{1 / 2}$ is the one-dimensional equivalent to the solution of Boussineq's problem which shifts the contact area vertically by a certain amount and forms the neck which is typical for adhesive contacts.

The total force per unit length $P$ is:

$$
\begin{aligned}
P & =\int_{-a}^{a} p_{\mathrm{s}}(x, a) \mathrm{d} x \\
& =\frac{E}{2 R} \int_{-a}^{a}\left(a^{2}-x^{2}\right)^{1 / 2}+\frac{C-a^{2} / 2}{\left(a^{2}-x^{2}\right)^{1 / 2}} \mathrm{~d} x .
\end{aligned}
$$

With the integrals given in eqns. (84) and (83) this becomes

$$
P=\frac{\pi E}{2 R} C \text {. }
$$

The rolling moment $M_{y}=\int x p_{\mathrm{s}}(x, a) \mathrm{d} x$ of this pressure distribution is of course zero. 


\section{A.2. The asymmetric pressure distribution}

We now consider a contact where the contact region is of the same size as in the symmetric case, but is shifted with respect to the centre of the cylinder by an amount $\xi$ (see fig. 9). Then, the deformation again follows eqn. (50) but the contact strip is a shifted interval:

$$
u_{z}(x)=\delta_{z}-\frac{x^{2}}{2 R}, \quad-a-\xi \leq x \leq a-\xi
$$

For the calculation of the pressure distribution it is convenient to have the contact area symmetric about the origin. In order to do so we introduce the variable

$$
X=x+\xi
$$

Then the deformation can be written as

$$
u_{z}(X)=\delta_{z}-\frac{(X-\xi)^{2}}{2 R}, \quad-a \leq X \leq a
$$

Introducing this result into eqn. (51) yields for the asymmetric pressure distribution $p_{\mathrm{a}}(X, a)$

$$
p_{\mathrm{a}}(X, a)=-\frac{E}{2 R\left(a^{2}-X^{2}\right)^{1 / 2}}\left[C+\frac{1}{\pi} \int_{-a}^{a} \frac{s\left(a^{2}-s^{2}\right)^{1 / 2}}{X-s} \mathrm{~d} s-\frac{\xi}{\pi} \int_{-a}^{a} \frac{\left(a^{2}-s^{2}\right)^{1 / 2}}{X-s} \mathrm{~d} s\right] .
$$

With eqns. (81) and (82) this can be written as

$$
p_{\mathrm{a}}(X, a)=\frac{E}{2 R}\left[\left(a^{2}-X^{2}\right)^{1 / 2}+\left(C-\frac{a^{2}}{2}+\xi X\right) \frac{1}{\left(a^{2}-X^{2}\right)^{1 / 2}}\right] .
$$

Thus the pressure distribution differs from the symmetric one only by an additional term which is proportional to $X$ and, therefore, not symmetric. As we will see, this term is responsible for the moment associated with this pressure distribution.

The total force associated with this distribution is again (see eqn. (57))

$$
P=\frac{\pi E}{2 R} C
$$

and the rolling moment about the $y$ axis which intersects the $x$ axis at $X=0$ is given by

$$
\begin{aligned}
M_{y}^{X=0} & =\int_{-a}^{a} X p_{\mathrm{a}}(X, a) \mathrm{d} X \\
& =\frac{E}{2 R} \int_{-a}^{a} \xi \frac{X^{2}}{\left(a^{2}-X^{2}\right)^{1 / 2}} \mathrm{~d} X \\
& =\frac{\pi a^{2} E}{4 R} \xi .
\end{aligned}
$$


We still have to transform this result back in the original coordinate system. The moment about the $y$ axis that intersects the $x$ axis at $x=0$ can be calculated by

$$
M_{y}^{X-\xi}=M_{y}^{X=0}-\xi P,
$$

and thus

$$
M_{y}^{x=0} \approx \frac{\pi}{4} \frac{E}{R} \xi\left(a^{2}+2 C\right) .
$$

This is the exact result which we will compare with the approximate calculation performed in the following section.

\section{A.3. Simplified calculation of the rolling moment}

The moment of the asymmetric distribution can also be approximately calculated in the following way. We assume that the $x<0$ half of the contact area has a pressure distribution equal to the symmetric distribution $p_{\mathrm{s}}(x, a+\xi)$ with contact strip half-width $a+\xi$, while the $x>0$ part of the contact area has a distribution equal to a symmetric distribution $p_{\mathrm{s}}(x, a-\xi)$, thus with respect to a contact half-width of $a-\xi$. The overall distribution calculated in this way is therefore not continuous at $x=0$.

The moment associated with this distribution is

$$
\begin{aligned}
M_{y} & =\int_{-a-\xi}^{0} x p_{\mathrm{s}}(x, a+\xi) \mathrm{d} x+\int_{0}^{a-\xi} x p_{\mathrm{s}}(x, a-\xi) \mathrm{d} x \\
& =-\int_{0}^{a+\xi} x p_{\mathrm{s}}(x, a+\xi) \mathrm{d} x+\int_{0}^{a-\xi} x p_{\mathrm{s}}(x, a-\xi) \mathrm{d} x \\
& =-M_{y, a+\xi}^{(1 / 2)}+M_{y, a-\xi .}^{(1 / 2)}
\end{aligned}
$$

Thus we have to solve integrals of the kind

$$
\begin{aligned}
& M_{y, \infty}^{(1 / 2)}=\int_{0}^{\infty} x p_{s}(x, \infty) \mathrm{d} x \\
& =\frac{E}{2 R}\left[\int_{0}^{\infty} x\left(\mathscr{A}^{2}-x^{2}\right)^{1 / 2} \mathrm{~d} x+\left(C-\frac{\mathscr{A}^{2}}{2}\right) \int_{0}^{\mathscr{\alpha}} \frac{x}{\left(\mathscr{A}^{2}-x^{2}\right)^{1 / 2}} \mathrm{~d} x\right] .
\end{aligned}
$$

The required integrals are given in eqns. (85) and (86). The result is

$$
M_{y, \mathscr{L}}^{(1 / 2)}=\frac{E}{2 R}\left(-\frac{\mathscr{A}^{3}}{6}+C \cdot \mathscr{A}\right) .
$$

The total moment is now

$$
M_{y}=-M_{y, a+\xi}^{(1 / 2)}+M_{y, a-\xi}^{(1 / 2)},
$$

which, to first order in $\xi / a$, is

$$
M_{y} \approx \frac{1}{2} \frac{E}{R} \xi\left(a^{2}-2 C\right) .
$$

Comparing this result to eqn. (70), we can see that eqn. (78) is smaller by a factor $\pi / 2$, but otherwise reproduces correctly the dependencies upon the properties of the cylinder and upon the displacement $\xi$. This seems to be a very satisfactory result. It justifies the use of the approximate calculation of the rolling moment. 


\section{APPENDIX B}

INTEGRALS USED

The following integrals have been used in the paper. We write them in terms of two abbreviations:

$$
\begin{aligned}
& W_{1}(r, a)=\left(a^{2}-x^{2}\right)^{1 / 2}, \\
& W_{2}(r, a)=\left(1-r^{2} / a^{2}\right)^{1 / 2} .
\end{aligned}
$$

B.1. Cauchy principal values of some singular integrals important in the two-dimensional problem

$$
\begin{aligned}
& \text { P.V. } \int_{-a}^{a} \frac{s\left(a^{2}-s^{2}\right)^{1 / 2}}{x-s} \mathrm{~d} s=\pi\left(x^{2}-\frac{1}{2} a^{2}\right) \\
& \text { P.V. } \int_{-a}^{a} \frac{\left(a^{2}-s^{2}\right)^{1 / 2}}{x-s} \mathrm{~d} s=\pi x .
\end{aligned}
$$

B.2. Non-singular integrals

$$
\begin{gathered}
\int_{-a}^{a} \frac{1}{W_{1}(r, a)} \mathrm{d} x=\pi, \\
\int_{-a}^{a} W_{1}(r, a) \mathrm{d} x=\frac{1}{2} \pi a^{2}, \\
\int_{0}^{a} x W_{1}(r, a) \mathrm{d} x=\frac{1}{3} a^{3}, \\
\int_{0}^{a} \frac{x}{W_{1}(r, a)} \mathrm{d} x=a, \\
\int_{0}^{a} r^{2} W_{2}(r, a) \mathrm{d} r=\frac{\pi}{16} a^{3}, \\
\int_{0}^{a} \frac{r^{2}}{W_{2}(r, a)} \mathrm{d} r=\frac{\pi}{4} a^{3} .
\end{gathered}
$$

\section{REFERENCES}

Anderson, H. L. (editor), 1981, Physics Vademecum (New York: AIP).

Ball, R. C., and WitTen, T. A., 1984, Phys. Rev. A, 29, 2966.

Brocklehurst, J. E., 1977, Phys. Chem. Carbon, 13, 145.

Chokshi, A., Tielens, A. G. G. M., and Hollenbach, D., 1993, Astrophys. J., 407, 806.

Derjaguin, B. V., Muller, V. M., and Toporov, Y. P., 1975, J. Coll. Interf. Sci., 53, 413.

EASTERl.ING, K. E., and ThÖLEN, A. R., 1972, Acta metall, 20, 1001.

Hodgman, C. D. (editor), 1949, Handbook of Chemistry and Physics, 31 st edition (Cleveland, OH: Chemical Rubber, Publishing Co.).

IsRaelaChVILI, J., 1992, Intermolecular and Surface Forces (London, San Diego: Academic). JOHNSON, K. L., 1989, Contact Mechanics (Cambridge, New York: Cambridge University Press). Johnson, K. L., Kendall, K., and Roberts, A. D., 1971, Proc, R. Soc. Lond., 324, 301.

Kantor, Y., and WitTen, T. A., 1984, J. Physique Lett., 45, L675.

Kendall, K., AlFORD, N. M., and BirChall, J. D., 1987, Nature, 325, 794.

Kendall, K., and Padget, J. C., 1987, J. Adhesion, 22, 39. 
Landman, U., LuedtKe, W. D., Burnham, N. A., and Colton, R. J., 1990, Science, 248, 454. Muller, V. M., Yushenko, V. S., and Derjaguin, B. V., 1979, J. Coll. Interf. Sci., 77, 91 ; 1982 , Ibid., 92, 92.

SAVkOOR, A. R., 1992, Fundamentals of Friction: Macroscopic and Microscopic Processes, edited by I. L. Singer and H. M. Pollock (Dordrecht, Boston, London: Kluwer), p. 111.

SPERLING, G., 1964, PhD Thesis, Technische Hochschule Karlsruhe, Karlsruhe.

THÖlÉN, A. R., 1992, Fundamentals of Friction; Macroscopic and Microscopic Processes, edited by I. L. Singer and H. M. Pollock (Dordrecht, Boston, London: Kluwer), p. 95.

TománeK, D., 1993, Scanning Tunnelling Microscopy III, edited by R. Wiesendanger and H.-J. Güntherodt (Berlin, Heidelberg, New York: Springer), chap. 11, p. 269.

Weidenschilling, S. J., Donn, B., and Meakin, P., 1988, The Formation and Evolution of Planetary Systems, edited by H. A. Weaver and L. Danly (Cambridge, New York, Melbourne, Sydney: Cambridge University Press).

Zisman, W. A., 1963, Ind. Engng. Chem., 55, 19. 
